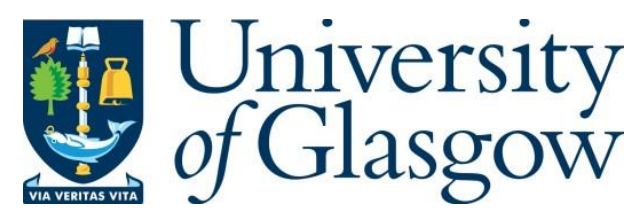

García Núñez, C., Vilouras, A., Navaraj, W. T., Liu, F. and Dahiya, R. (2018) ZnO nanowires based flexible UV photodetector system for wearable dosimetry. IEEE Sensors Journal, 18(19), pp. 7881-7888.

There may be differences between this version and the published version. You are advised to consult the publisher's version if you wish to cite from it.

http://eprints.gla.ac.uk/164777/

Deposited on: 3 July 2018

Enlighten - Research publications by members of the University of Glasgow http://eprints.gla.ac.uk 


\title{
ZnO Nanowires Based Flexible UV Photodetector System for Wearable Dosimetry
}

\author{
Carlos García Núñez, Anastasios Vilouras, William Taube Navaraj, Fengyuan Liu and Ravinder Dahiya \\ School of Engineering, University of Glasgow, G12 8QQ Glasgow, UK \\ Corresponding to: Ravinder.dahiya@glasgow.ac.uk
}

\begin{abstract}
This paper presents a flexible ultraviolet (UV) photodetector (PD) system based on zinc oxide (ZnO) nanowires (NWs) for wearable UV dosimetry. High-crystal quality ZnO NWs have been synthesized by chemical vapour transport (CVT) technique on $c$-plane sapphire substrates, and thereafter, transferred and aligned at pre-defined locations on a flexible substrate using dielectrophoresis (DEP). The accurate control over DEP parameters permitted the fabrication of large-area (wafer scale) arrays of ZnO NWs based UV PDs. Resulting PDs showed photocurrent-to-dark current ratios above $10^{3 \%}$, fast response times ( $<1 \mathrm{~s})$, high sensitivity to different $\mathrm{UV}$ light intensities, and good stability under several UV/dark irradiation cycles. In addition, above PDs presented a robust response under extreme bending conditions, which is critical for their application in high-performance wearable UV dosimeters.
\end{abstract}

Keywords- ZnO Nanowires, UV Photodetectors, Wearable Electronics, Optoelectronics, Dielectrophoresis, Nanowire Assembly

\section{INTRODUCTION}

There is a growing interest in wearable healthcare systems such as wearable pads and wrist-bands [1-3], for measurement of heart rate, blood pressure, electrocardiogram, sweat $\mathrm{pH}$, respiration rate, etc. [4-8] The field has opened several new application opportunities and is a major driver advancing the current rigid electronics towards the one with flexible and conformable form factors [9, 10]. For example, tattoo like sensing patches with several sensors and electronic components have been developed on flexible/stretchable substrates to allow conformal contact with the curvy surfaces of human body and thus to have reliable measurement of the physiological parameters [4, 9, 11-15]. Likewise, large-area robotic tactile sensing and corresponding electronics/interconnects have been developed on flexible substrates $[13,14,16]$.

One parameter of interest that has not been considered in wearable systems so far is the exposure of human body to the harmful light, with wavelengths $(\lambda)$ in the electromagnetic spectrum, i.e. the ultraviolet (UV) light. The UV light is around $3-5 \%$ of the total sun light received on the Earth surface [17]. About $95 \%$ of this UV light received on the Earth is type A (315 $<\lambda<400 \mathrm{~nm})$ and the rest comprises of type $\mathrm{B}(280<\lambda<315$ $\mathrm{nm})$, type $\mathrm{C}(100<\lambda<280 \mathrm{~nm})$ and Extreme UV namely EUV $(10<\lambda<121 \mathrm{~nm})$, mainly because of the ozone layer blocking effect. The controlled exposure to UV light can lead to several health benefits, including an enhancement of our mood (higher levels of a beta-endorphin molecule activating feel-good processes in the brain) and an increase of vitamin D levels, as well as, skin treatments (psoriasis, eczema, dermatitis, etc.) [17].

An earlier version of this paper was presented at the IEEE Sensors Conference 2017 (Glasgow, UK) and published in its Proceedings (DOI: 10.1109/ICSENS.2017.8234428)
As a result, deliberate tanning is often performed to develop a natural blocking layer based on pigment melanin, which absorbs both UVA and UVB [17]. In contrast, the excessive sun light exposure can also have several health risks, which include accelerated skin aging and the promotion of skin cancers such as basal cell carcinoma, squamous cell carcinoma, and malignant melanoma [18-20]. The UV can also damage eyes, immune system and can lead to skin burn and cancer. UVA can cause irreversible damage to our DNA, and both UVA and UVB radiations can dramatically destroy vitamin A in the skin [21, 22]. While sunblock lotions have been developed for protection against UVB, there are not many solutions for UVA $(315<\lambda<$ $400 \mathrm{~nm})[23]$. The above health benefits and potential risks make it important to monitor the exposure of human body to UV light specially in the UVA wavelength range (i.e. $\lambda<400 \mathrm{~nm}$ ).

Here, we present a flexible UV photodetector (PD) system based on zinc oxide $(\mathrm{ZnO})$ nanowires $(\mathrm{NWs})$. The presented PDs show $>10^{3} \%$ photocurrent-to-dark current ratios, fast response times $(<1 \mathrm{~s})$, high sensitivity to various UV light intensities, and excellent stability under several UV/dark irradiation cycles. The devices have been tested under extreme bending conditions, and the robust response justifies their suitability for high-performance wearable UV dosimetry application. This paper extends the work presented at the IEEE Sensors Conference 2017 [24]. The new inputs include: (i) detailed investigation about chemical vapour transport (CVT) technique, the influence of growth parameters (e.g. gas precursor flow, position of growth substrate, etc.), and growth substrate orientation with respect to the precursor gas direction, on the resulting morphology and aspect-ratio of $\mathrm{ZnO} \mathrm{NWs}$; (ii) the DEP assembling mechanism of NWs with lengths shorter than the gap between pair of conductive electrodes; (iii) analysis of UV PDs based on ZnO NWs under tensile and compressive bending; (iv) experimental study and theoretical modelling of PDs photoresponse and response time; (v) scaling up the PDs, with fabrication of $2 \times 2$ and $4 \times 4$ arrays on large-area (i.e. wafer scale) flexible substrates.

The paper is organised as follows: Section II presents experimental details, including material selection, a description of the CVT system, growth parameters and series of growths investigated in this work. Section III presents the characterization of ZnO NWs morphology, their DEP assembly between conductive electrodes and over large-area flexible substrates, the experimental characterization and modelling of the PD response under UV illumination, and the PD performance under different bending conditions. Finally, the key outcomes and future works are summarised in Conclusions. 


\section{EXPERIMENTAL DETAILS}

\section{A. Material Selection}

Ideally, UV PDs should be sensitive in the UV region and should have high signal-to-noise ratio (SNR), selectivity, photoresponsivity and fast response $[25,26]$. In this regard, $\mathrm{ZnO}$ is attractive as its wide energy band gap $\left(E_{\mathrm{g}}=3.3 \mathrm{eV}\right)$ results in the low absorption in the visible range and thus allows high UV sensitivity and selectivity. Photoconductors based on bulk $\mathrm{ZnO}$ have been reported in literature [27]. The charge trapping effects at surface states and grain boundaries in these cases lead to long generation to recombination times, which eventually results in high photoconductive gains but also slows down the optical response. This can be overcome by using PDs based on $\mathrm{ZnO}$ NWs, whose high surface-to-volume ratio could help attain PDs with highest gain values among all the structures and materials with UV sensitivity. Further, the use of NWs can allow PDs on flexible substrates. UV PDs on flexible substrates have distinct advantage with respect to their rigid counterparts as they could be in conformal contact with the body and can be used to monitor the optimal UV dose received from the sun light. Their cost-effective manufacturing, excellent mechanical properties and compatibility with Si technology are also useful [28, 29]. Surprisingly, the flexible UV PD based on ZnO NWs has rarely been investigated and this paper addresses this gap [30,31]. The main challenge is to fabricate $\mathrm{ZnO}$ NWs on flexible substrates as the thermal budget issue (i.e. incompatibility between the high temperature steps needed for NW synthesis and melting temperature of flexible substrates) makes it difficult. The low temperature methods for synthesis of $\mathrm{ZnO} \mathrm{NWs}$ explored in literature are not attractive as they result in poor quality nanostructures, which eventually result in devices with poor performance [31]. The thermal budget issue has been overcome in this paper by splitting the fabrication steps on the basis of temperature. The high-quality ZNO NWs, which require hightemperature $\left(\sim 950^{\circ} \mathrm{C}\right)$ are obtained by CVT in a quartz tube, as thoroughly explained in Sec II.B. These high-quality NWs are then transferred to flexible substrates with pre-patterned electrodes, as explained further in Sec II.C.

\section{B. Synthesis of Nanowires}

The NW synthesis by either top-down or bottom-up approach, with accurate control over crystalline structure and uniform aspect ratio is an active area of intensive research [28, $32,33]$. The quality of as-grown NWs is crucial for the performance of the transfer process and later on for the overall device performance. $\mathrm{ZnO} \mathrm{NWs}$ were synthesized on $c$-plane sapphire substrates by CVT in a quartz tube placed inside a high temperature horizontal furnace (Fig. 1). The NW synthesis is based on Au-assisted vapour-liquid-solid (VLS) approach [11]. Firstly, a 5-nm thick Au layer was evaporated on top of a sapphire substrate and annealed at $1050^{\circ} \mathrm{C}$ for $15 \mathrm{~min}$ in $\mathrm{Ar}$ ambient, resulting in a random distribution of $\mathrm{Au}$ NPs. In our CVT process, $\mathrm{ZnO}$ micro-powder $(<5 \mu \mathrm{m}$ particle size, $3 \mathrm{~N})$ was used as $\mathrm{Zn}$ source; that $\mathrm{ZnO}$ powder was mixed with graphite (C) powder $(<20 \mu \mathrm{m}$ particle size) resulting in a $1 \mathrm{~g}$ mixture with a $\mathrm{ZnO}: \mathrm{C}$ mass ratio of 1:1. Then, the mixture and sapphire substrate were loaded in a ceramic crucible, which was transferred to the centre of the quartz tube, at room temperature. To analyse the effect of substrate orientation with respect to the precursor gases flow on the resulting $\mathrm{NW}$ properties, the $c$ -

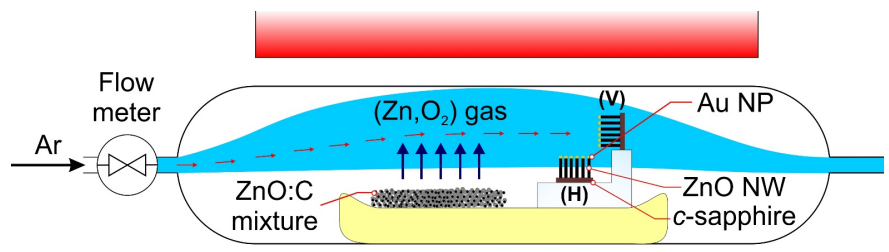

CVT system

Fig. 1. 2D schema of CVT system and growth of ZnO NWs by Au-assisted VLS approach on $c$-plane sapphire substrates loaded $(\mathrm{H})$ horizontally and (V) vertically with respect to the gas flow.

sapphire substrates were loaded either in horizontal (" $\mathrm{H}$ " in Fig. 1) or vertical positions ("V" in Fig. 1), keeping a distance of around $1 \mathrm{~cm}$ with respect to the $\mathrm{ZnO}: \mathrm{C}$ powder mixture. Each growth, i.e. using different substrate orientation, has been carried out in different process to ensure the same experimental conditions (i.e. gas content, residual pressure, gas flow, etc.), and therefore, to guarantee the reproducibility of the growth process.

The CVT process was carried out at $950^{\circ} \mathrm{C}$, leading to the carbothermal reduction of $\mathrm{ZnO}$ powder, producing $\mathrm{Zn}(\mathrm{g})$ and oxygen gas species to the ambient $[28,34]$. Both $\mathrm{Zn}(\mathrm{g})$ and $\mathrm{O}_{2}(\mathrm{~g})$ were carried towards the sapphire substrate surface by using different Ar fluxes ranging from 100 to $1000 \mathrm{~cm}^{3} / \mathrm{min}$, where VLS growth of ZnO NWs takes place. In this scenario, $\mathrm{Zn}(\mathrm{g})$ and $\mathrm{O}_{2}(\mathrm{~g})$ could be absorbed at $\mathrm{Au}$ liquid nanodroplets. When these droplets become supersaturated, there is a precipitation of $\mathrm{ZnO}(\mathrm{s})$ towards the interface formed between the $\mathrm{Au}$ droplet and the sapphire substrate surface, and this eventually leads to the nucleation of $\mathrm{ZnO} \mathrm{NW}$ under the $\mathrm{Au}$ droplet. With NW like crystal accumulating enough tension, the $\mathrm{Au}$ droplet is lifted up by the NW. If VLS conditions are preserved during the NW growth (i.e. $\mathrm{Zn}(\mathrm{g})$ and $\mathrm{O}_{2}(\mathrm{~g})$ fluxes at the $\mathrm{Au} / \mathrm{ZnO}$ interface), the $\mathrm{NW}$ can reach lengths from few nanometers to hundreds of microns.

\section{Assembly of Nanowires}

The small dimensions of NWs (diameters below $100 \mathrm{~nm}$ and lengths from few nanometers and to hundreds of microns) present a challenge for their integration onto large areas and nonconventional flexible/stretchable substrates [35]. In this regard, a number of techniques has been investigated to transfer NWs from the growth substrate to the receiver substrate, with controlled location and print area, NW-to-NW spacing and NW surface/linear density and preserving as-grown NWs. Some of these techniques, including transfer printing, contact-printing, roll-printing, Langmuir-Blodgett, and DEP, have already demonstrated their potential to produce uniform electronic layers based on different semiconductor NWs [25, 36-41]. These techniques aim to reduce the fabrication cost while addressing thermal budget issue, enhancing the NW transfer yield and improving the process reproducibility. DEP is a well-known technique which has been successfully used to align NWs, nanoparticles, and carbon nanotubes (CNTs) on different kinds of substrates [25, 36-39]. The technique relies on non-uniform electric fields to trap and to align NWs in solution, at specific sites over the receiver substrate, at room-temperature, and without need of expensive tools. DEP technique can control not only the resulting location of the NWs on the receiver substrate, 
(a)

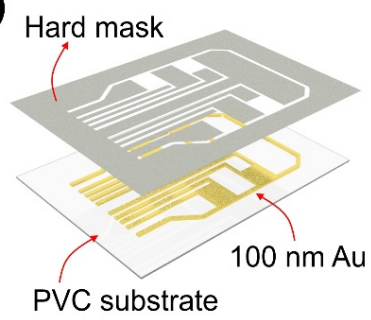

(b)

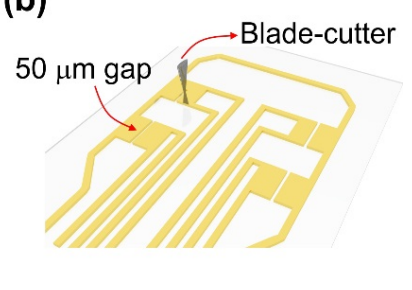

(d)

(c)

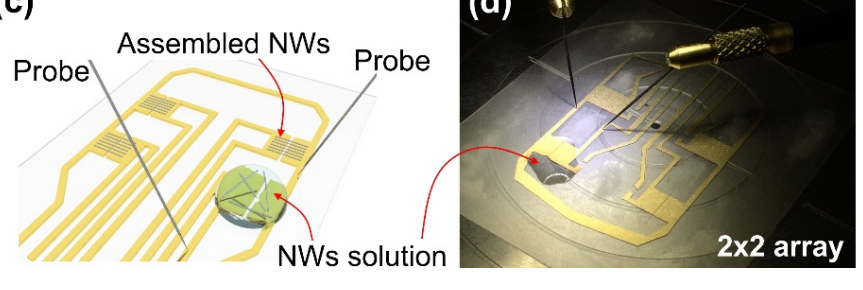

Fig. 2. (a-c) 3D schema of PD fabrication steps: (a) electrodes patterning by hard-masking; (b) electrodes gap patterning by blade-cutting; (c) NWs solution drop-casting and DEP assembling of $\mathrm{ZnO}$ NWs. (d) Image of $\mathrm{ZnO} \mathrm{NWs}$ assembled by DEP between Au electrodes, forming a $2 \times 2$ array of UV PDs.

but also the number of trapped NWs through the use of specific electrode geometries [25]. Accordingly, DEP has been used here to assemble NWs (Fig. 2). Due to dimensional variability, it is still challenging to obtain response uniformity among single NW based devices spread over large areas. This issue could be solved by having devices made from ensemble of NWs. The lesser statistical variation in the dimensions of ensemble of NWs is likely to lead to a uniform response from different devices distributed on a flexible substrates [41, 42]. For this reason, we have used ensemble of NWs to realize the PDs.

Using a hard mask, 100-nm thick Au electrodes were evaporated with electron-beam on a 40- $\mu \mathrm{m}$ thick polyvinyl chloride (PVC) flexible substrate (Fig. 2(a)). A 50- $\mu$ m width gap was defined between electrodes by blade-cutting (Fig. 2(b)) and this was repeated to obtain $2 \times 2$ and $4 \times 4$ arrays of gaps. Following this, the $\mathrm{ZnO}$ NWs were assembled by DEP on a PVC substrate with pre-patterned pairs of Au electrodes (Fig. 2(c)). Prior to DEP, $\mathrm{ZnO}$ NWs were transferred from the growth substrate to a $25-\mathrm{ml}$ volume of ethanol solution by using a probe sonicator ( $100 \mathrm{~W}$ for $30 \mathrm{~s}$ ). A $200-\mu \mathrm{l}$ volume droplet of the NW solution was drop-casted on top of each gap in the electrodes array while applying an AC signal (Fig. 2(d)). For these experiments, we have used DEP parameters previously optimized for $\mathrm{ZnO}$ NWs solutions with similar concentrations [7], consisting in an $\mathrm{AC}$ signal with a peak-to-peak voltage $\left(V_{\mathrm{pp}}\right)$ of $15 \mathrm{~V}$ and a frequency $\left(f_{\mathrm{AC}}\right)$ of $1 \mathrm{MHz}$. In DEP, the nonuniform electric field is applied through the NWs in solution to create local dipoles along the nanostructures and to attract them towards electrodes gap by taking advantage of the electric field gradient [13].

\section{RESULTS AND DISCUSSION}

\section{A. Characterization of $\mathrm{ZnO}$ Nanowires}

The effect of both Ar flow and the substrate orientation with respect to $\mathrm{Ar}$ gas flow direction (Fig. 1) on the resulting $\mathrm{ZnO}$ NW properties (morphology, aspect ratio, crystallinity) has been thoroughly analysed here. The horizontal substrates showed a clear non-uniformity of the resulting NW lengths

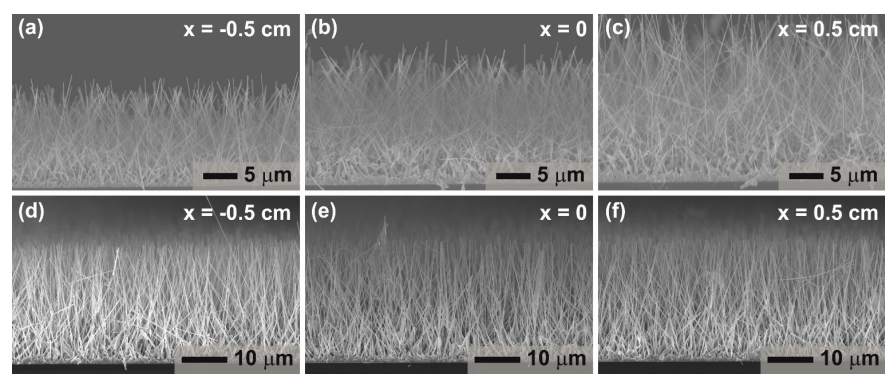

Fig. 3. SEM images of CVT ZnO NWs grown on $c$-sapphire substrates (a-c) horizontally and (d-f) vertically aligned with respect to the gas flow direction.

along the sample surface for CVT growths carried out for $1 \mathrm{~h}$ and using an Ar flow of $500 \mathrm{~cm}^{3} / \mathrm{min}$ (Fig. 3(a-c)). Accordingly, we have analysed by SEM the centre of a $1 \times 1 \mathrm{~cm}^{2}$ sample (Fig. 3(b)), and another two areas $0.5 \mathrm{~cm}$ away the centre (Fig. 3(a, c)). This characterization shows NWs that are $\sim 27 \mu \mathrm{m}$ long at the central area of analysis (Fig. 3(b)). In the areas closer (Fig. 3(c)) and farther to the $\mathrm{ZnO}: \mathrm{C}$ mixture during CVT growth (Fig. 3(a)), the NW lengths were $\sim 19$ and $33 \mu \mathrm{m}$, respectively. The horizontal position of the substrate with respect to the gas flow clearly leads to a non-uniform concentration of the precursor gas along the substrate, resulting in a non-uniform NW growth rates along the sample. This drawback was partially overcome by increasing the Ar gas flow. Accordingly, a series of experiments were carried out with Ar flows ranging between 100 to $1000 \mathrm{~cm}^{3} / \mathrm{min}$. The outcome was a significant improvement in terms of the uniformity of NW lengths. However, this was not free from side-effects, as the increase of Ar flow also produced an uncontrolled nucleation of a parasitic crystalline layer on the substrate surface. The likely fallout of this side-effect is the poor control over the conditions for VLS, particularly for long CVT growths, which could affect the morphology and crystalline structure of the NWs.

When the substrate was placed vertically with respect to the gas flow, we noted a significant improvement in the NW uniformity over all the investigated areas (Fig. 3(d-f)). The average NW length obtained in these conditions was $\sim 27 \mu \mathrm{m}$ for $1 \mathrm{~h}$ CVT growth with an Ar flow of $500 \mathrm{~cm}^{3} / \mathrm{min}$, i.e. a growth rate of around $0.45 \mu \mathrm{m} / \mathrm{min}$. For the sake of reproducibility, NWs shown in Fig. 3(d-f) were used for fabricating UV PDs by DEP. The structural, compositional and optical characterizations of as-grown $\mathrm{ZnO}$ NWs have shown that they have high crystal quality, a stoichiometric composition (i.e. $\mathrm{Zn}_{\mathrm{x}} \mathrm{O}_{1-\mathrm{x}}, \mathrm{x} \sim 0.5$ ), and an absorption edge at $\lambda$ $\sim 380 \mathrm{~nm}$. Accordingly, these NWs exhibited a direct wide energy band gap $\left(E_{\mathrm{g}}\right)$ of around $3.23 \mathrm{eV}$, demonstrating their validity as building block in UV PDs [24].

\section{B. Dielectrophoretic Assembly of Nanowires}

The DEP process has been utilized here to fabricate UV PDs over large-area flexible substrates. The cost-effective fabrication steps developed in this work, allowed us to fabricate both $2 \times 2$ and $4 \times 4$ arrays of UV PDs based on $\mathrm{ZnO} \mathrm{NWs}$, demonstrating the scalability of the method. The resulting assembly of ZnO NWs was characterized by SEM (Fig. 4(a)) 


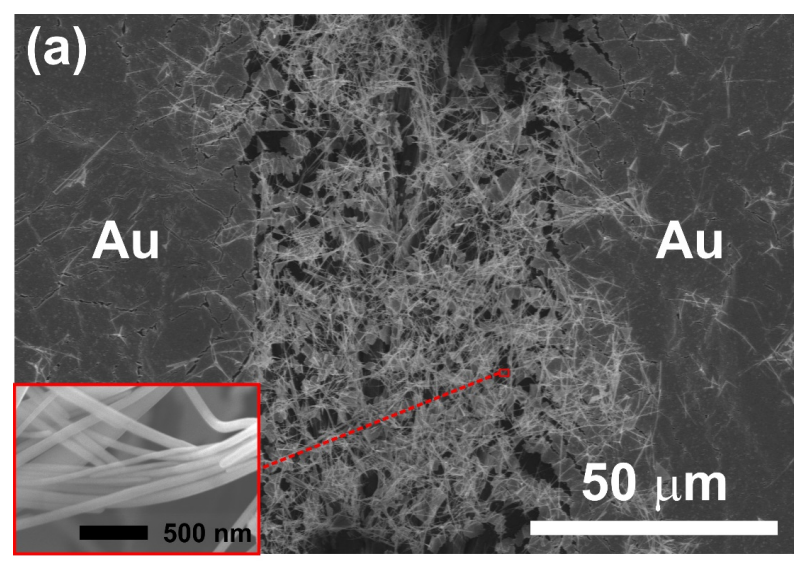

(b)

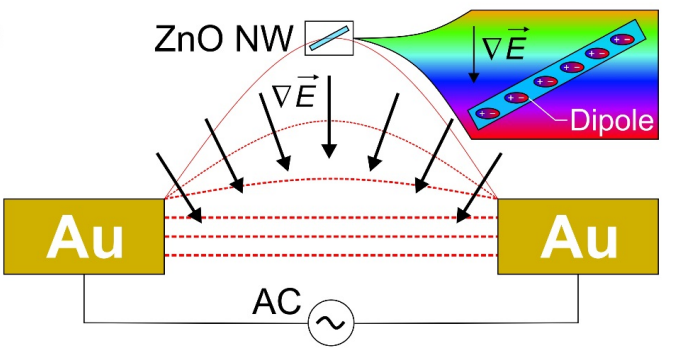

(c)

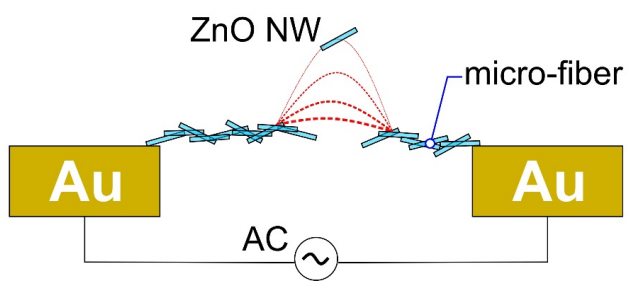

Fig. 4. (a) SEM image of CVT ZnO NWs, DEP assembled between $\mathrm{Au}$ electrodes. Inset: micro-fibre based on $\mathrm{ZnO}$ NWs, bridging Au electrodes. 2D schema of DEP process, including (b) the formation of a $\nabla \boldsymbol{E}$ in the NWs solution, inducing local dipoles along the NW structure; (c) trapping of NWs at high electric field regions, resulting in a micro-fibre consisting of a $\mathrm{ZnO} \mathrm{NWs}$ interlaced.

and the formation of a micro-fibre consisting of a $\mathrm{ZnO}$ NWs interlaced bridging a pair of Au electrodes was observed (see inset of Fig. 4(a)). In contrast to conventional DEP, where the spacing between electrodes is less than the length of the NWs in solution [25], here, we have successfully used a gap of around $50 \mu \mathrm{m}$-defined by blade-cutting- and assembled with DEP the NWs having lengths around $30 \mu \mathrm{m}$. Since the gap is larger than the NWs length, the DEP process comprises: (i) dipoles formation along NWs structure induced by the electric field (Fig. 4(b)); (ii) NWs trapping at high electric field regions along the gradient of the electric field $(\nabla \boldsymbol{E})$; (iii) the propagation of electric field through assembled NWs (Fig. 4(c)); (iv) microfibre formation bridging the gap between electrodes. The assembly of nanostructures with a dimension smaller than the electrodes gap was successfully demonstrated in $\mathrm{Au}$ nanoparticles [43]. However, our process prevents the use of lithography steps to define the electrodes gap and involves the use of low amplitude $\mathrm{AC}$ signals $\left(V_{\mathrm{pp}}=15 \mathrm{~V}\right.$ and $\left.f_{\mathrm{AC}}=1 \mathrm{MHz}\right)$, which prevents any potential damage of the NW properties caused during the assembling process.

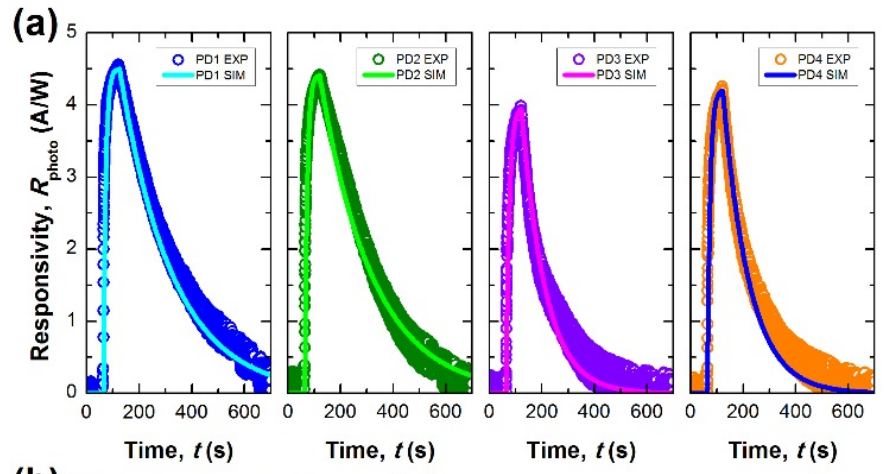

(b)

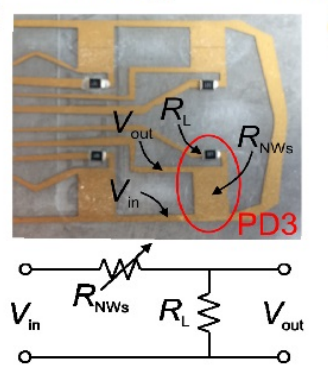

(c)

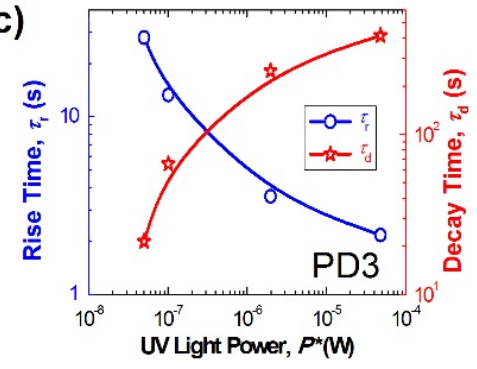

Fig. 5. (a) $R_{\text {photo }}$ vs. time for PD1-4, using a $V_{\text {in }}=5 \mathrm{~V}, R_{\mathrm{L}}=100 \mathrm{M} \Omega$, and a $P^{*}=$ $1 \mu \mathrm{W}$. (b) Image of $2 \times 2$ UV PDs array (top) and equivalent circuit diagram (bottom). (c) $\tau_{\mathrm{r}}$ and $\tau_{\mathrm{d}} \mathrm{vs.} P^{*}$ for PD3.

\section{Characterization and Modelling of UV PDs Photoresponse}

The photocurrent $\left(I_{\text {photo }}=I_{\text {light }}-I_{\text {dark }}\right)$ of each PD in a $2 \times 2$ sensing array (i.e. PD1-4) was measured by using a UV lightemitting diode (LED) $(365<\lambda<370 \mathrm{~nm}$ and an optical power of $200 \mathrm{~mW}$ at $700 \mathrm{~mA})$. Fig. 5(a) shows the experimental photoresponsivity $\left(R_{\text {photo }}=I_{\text {photo }} / P^{*}\right)$ of each PD measured overtime, using a low intensity UV light power $\left(P^{*}\right)$ of $1 \mu \mathrm{W}$ (i.e. a power density of $\left.1 \mu \mathrm{W} / \mathrm{cm}^{2}\right)$. The characterization of PDs was carried out using a probe station in a Faraday cage to prevent the influence of any electromagnetic noise and to ensure controlled light and air ambient conditions. The well-known sensitivity of $\mathrm{ZnO}$ nanomaterials to gases in the ambient [44], could hinder the characterization for UV response. For the sake of reproducibility and reliability of the measurements, both relative humidity $(\mathrm{RH})$ and temperature of the ambient were monitored by using a digital hygrometer (from Farnell, HTD-625). In this work, $\mathrm{RH}$ of $20 \%$ and a temperature of $25^{\circ} \mathrm{C}$ were measured during the characterization of the PDs.

The PD current $\left(I_{\mathrm{PD}}=V_{\text {out }} / R_{\mathrm{L}}\right)$ was measured by utilizing the circuit presented in Fig. 5(b), where $R_{\mathrm{L}}$ is the load resistance $(100 \mathrm{M} \Omega), V_{\text {out }}$ is the output voltage measured by an Arduino Uno board (sampling rate of $100 \mathrm{~ms}$ ), and $V_{\text {in }}$ is the input voltage $(5 \mathrm{~V})$. From Fig. 5(a), it can be concluded that the PDs exhibited a maximum $R_{\text {photo }}$ around $4.3 \pm 0.1 \mathrm{~A} / \mathrm{W}$, confirming the validity of the fabrication method to produce a PD arrays with uniform $R_{\text {photo }}$ in the UV light range (i.e. $365<\lambda<370 \mathrm{~nm}$ ). The resulting improvement in response uniformity can be attributed to the DEP assembly of NWs forming an UV sensitive micro-fibre. Fig. 5(a) represents experimental data (EXP) along with the simulation results (SIM) obtained by Cadence Virtuoso environment. Based on the exponential response of the PD in dark $(t<60 \mathrm{~s})$ and under UV illumination $(t>60 \mathrm{~s})$ the proposed PD behavioural model was implemented in Verilog-A and 
TABLE I. COMPARISON OF RESPONSIVITY OBTAINED FROM UV PHOTODETECTORS BASED ON NANOSTRUCTURES.

\begin{tabular}{cccc}
\hline \hline UV Sensing Material & $\begin{array}{c}\boldsymbol{R}_{\text {photo }} \\
(\mathbf{A} / \mathbf{W})\end{array}$ & $\begin{array}{c}\boldsymbol{P} \\
\left(\mathbf{m W} / \mathbf{c m}^{2}\right)\end{array}$ & Ref. \\
\hline Multiple $\mathrm{ZnO} \mathrm{NWs}$ & 4.3 & 0.001 & This \\
$\mathrm{ZnGa}_{2} \mathrm{O}_{4} \mathrm{NWs}$ & 3174 & 0.53 & {$[52]$} \\
$\mathrm{TiO}_{2} \mathrm{NTs}_{2}$ & 78.3 & 10 & {$[48]$} \\
$2 \mathrm{D} \mathrm{Bi}_{2} \mathrm{Te}_{3}-\mathrm{SnS}_{-} \mathrm{Bi}_{2} \mathrm{Te}_{3}$ & 115 & 10 & {$[47]$} \\
$2 \mathrm{DoS}_{2}-\mathrm{CQD}$ & 8.4 & 3.1 & {$[51]$} \\
Perovskite and conjugated polymer & 0.011 & 0.5 & {$[53]$} \\
Organolead Halide Perovskite & 3.49 & 0.21 & {$[54]$} \\
$\mathrm{GaS} \mathrm{NS}_{\mathrm{MoS}} \mathrm{NS}$ & 19.2 & 0.5 & {$[49]$} \\
& 183 & 200 & {$[50]$}
\end{tabular}

NWs: nanowires; NTs: nanotubes; CQD: carbon quantum dot; 2D: two-dimensional material; $P$ : power density of UV light source; NS: Nanosheet; CNT: carbon nanotube.

compiled in Cadence Virtuoso as a voltage controlled current source (VCCS). The $I_{\text {dark }}$ and $I_{\text {light }}$ measured from each PD, were simulated by applying a square wave pulse acting as dark and light sources, respectively. In this scenario, the $I_{P D}$ was modelled by [25]

$$
I_{\mathrm{PD}}=G\left(1-\mathrm{e}^{-t / \tau}\right)
$$

Where $G$ is the gain of the VCCS which modulates the maximum $I_{\mathrm{PD}}$ obtained by each of the four PDs, and $\tau$ is the time constant - namely rise time $\left(\tau_{\mathrm{r}}>0\right)$ and decay time $\left(\tau_{\mathrm{d}}<0\right)-$ characteristic of each PD. With suitable assumptions, the above model can be extended to simulate device response under various bending conditions $[45,46]$, as discussed in next subsection.

The simulated $R_{\text {photo }}$ was fitted to the experimental data (Fig. 5(a)) to calculate both $\tau_{\mathrm{r}}$ and $\tau_{\mathrm{d}}$. Fig. 5(c) present $\tau_{\mathrm{r}}$ and $\tau_{\mathrm{d}}$ as a function of $P^{*}$ for PD3. It was observed that the time constants of the fabricated PD3 are strongly affected by $P^{*}$, exhibiting a decrease of $\tau_{\mathrm{r}}$ and an increase of $\tau_{\mathrm{d}}$ with $P^{*}$. This is in good agreement with previously reported works [25]. As mentioned above, the photo-responsivity of $\mathrm{ZnO} \mathrm{NWs}$ is mainly governed by surface traps [25]. On one hand, the increase of the UV light power produces more number of photons irradiating the NW structure, releasing charges trapped at the surface of the NW to the conductive volume and photo-generating more electrons in the NW bulk. Both effects lead to a lower $\tau_{\text {r. }}$ On the other hand, the $I_{\text {dark }}$ of a $\mathrm{ZnO} \mathrm{NW}$ is mainly given by the surfaces traps of the NW, i.e. the high resistivity of the NWs is produced by the gas molecules of the ambient (mainly oxygen-based gases) trapping free charges from the NW bulk at the surface of the NW. In this regard, the re-absorption of the oxygen molecules at the NW surface, after the UV illumination to recover the initial $I_{\text {dark, }}$ will be hindered (i.e. longer $\tau_{\mathrm{d}}$ ) by increasing the UV light power. This means more number of oxygen molecules are desorbed during the illumination of the NW.

Although the fabrication steps of each PD in the $2 \times 2$ array are same, the DEP process can result in different number of assembled NWs or connections between NWs. Moreover, the non-uniformity of the NW size in the solution can hinder the control over each PD performance. For these reasons, the $R_{\text {photo }}$ and response time of each PD in the array show slight variations

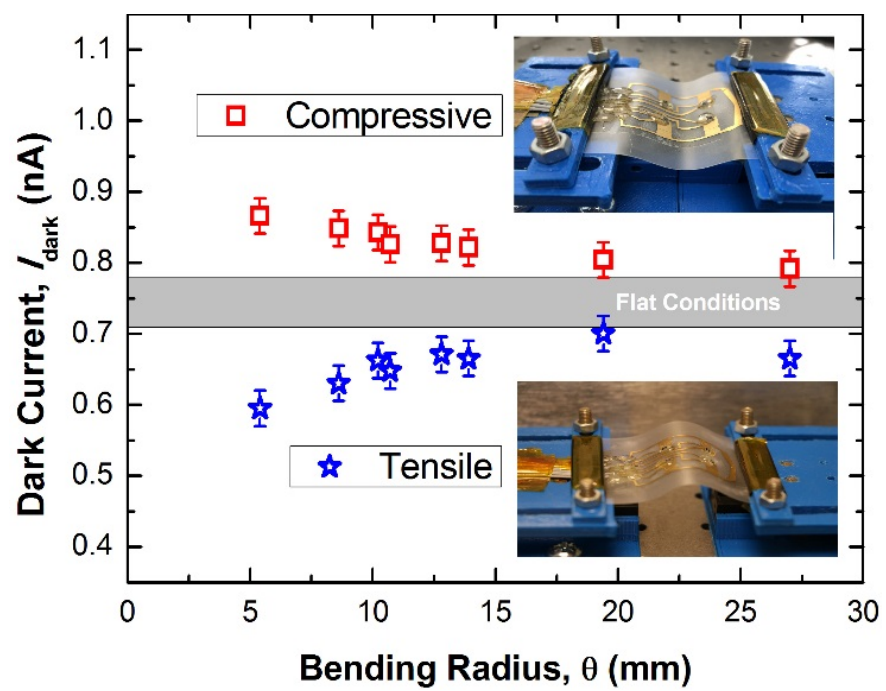

Fig. 6. $I_{\text {dark }}$ vs. $\theta$ of UV PD1-4, measured under both compressive and tensile stress. Inset: snapshot of $2 \times 2$ UV PDs array taken during dynamic bending.

$\left(\tau_{\mathrm{r}}=12 \pm 2 \mathrm{~s}, \tau_{\mathrm{d}}=230 \pm 100 \mathrm{~s}, I_{\mathrm{photo}} / I_{\mathrm{dark}}=2027 \pm 200 \%\right)$. However, these variations can be easily normalized, allowing to evaluate the area distribution of the light intensity received by the large area PD from different kinds of UV sources. PDs performance shows a high UV $I_{\text {photo }} / I_{\text {dark }}$ above $10^{3} \%$ and a moderate maximum $R_{\text {photo }}$ of 4.5 , mainly due to the use low light power $\left(\sim \mu \mathrm{W} / \mathrm{cm}^{2}\right)$. Here, the slow recovery time observed in all PDs is not a drawback since fabricated devices are meant to be used as UV dosimeter, where total dose received by a specific surface is the crucial factor. The response stability of PD1-4 is confirmed by observing similar SNR after multiple dark/UV cycles (not show).

Compared to the high-performance UV PDs based on other nanomaterials and structures (Table I), including twodimensional (2D) semiconductor heterostructures [47], metal oxide nanotubes (NTs) [48], semiconductor nanosheets (NS) [49, 50], 2D materials combined with carbon quantum dots (CQD) [51], ternary compound based NWs [52], and organic materials $[53,54]$, the UV PDs fabricated in this work highlight due to their high responsivity to low light source intensities. For example, the UV PDs developed in this work present $R_{\text {photo }}$ around $4.3 \mathrm{~A} / \mathrm{W}$ which are similar than those obtained by $2 \mathrm{D}$ $\mathrm{MoS}_{2}-\mathrm{CQD}$, the latter using a light source with a power density three orders of magnitudes higher than the one used here (Table I). Furthermore, comparing single-NW and multi-NW based UV PDs, the latter shows lower $R_{\text {photo }}$ due to a faster recombination time caused by multiple NWs junctions [25]. In contrast, multiNWs UV PDs demonstrate compatibility to flexible substrates, presenting great stability and uniform densities at large sensing areas, mainly due to the statistical compensation of NWs with different diameters, averaging the response of the PD. Furthermore, the great sensitivity of PDs presented here to low UV irradiation intensities $\left(1 \mu \mathrm{W} / \mathrm{cm}^{2}\right)$ is close to record values obtained in flexible UV PDs also based on $\mathrm{ZnO}$ nanomaterials [55] which probe not only their applicability in flexible electronics but also to the detection of low UV doses.

Although organic materials present also great compatibility with flexible substrates, their low $R_{\text {photo }}$ obtained even at high 

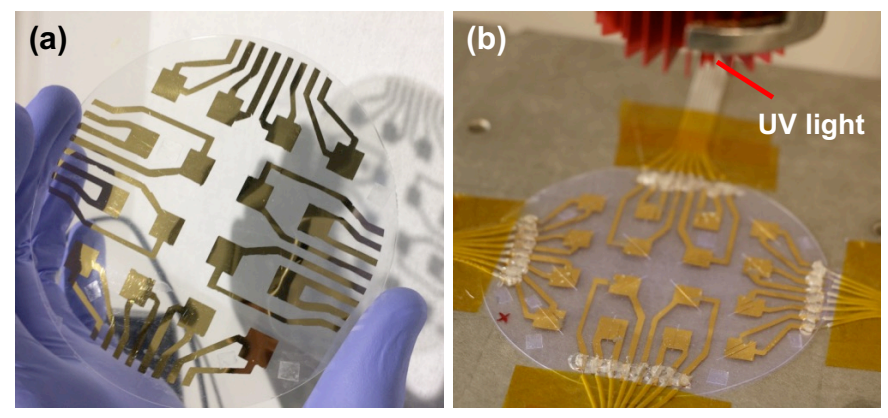

(c)

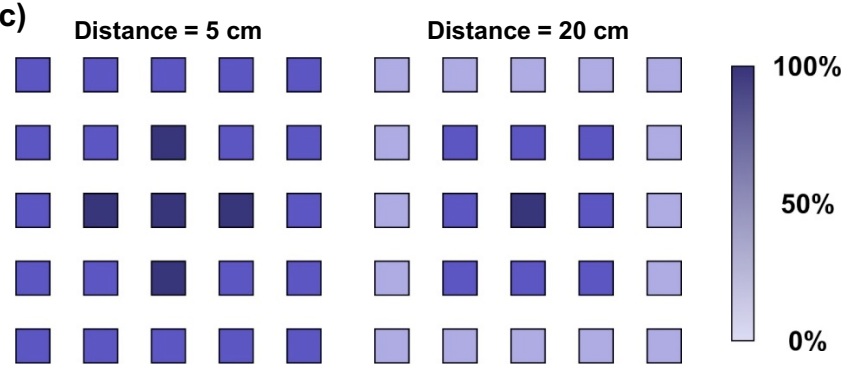

Fig. 7. (a) $4 \times 4$ array of UV PDs based on $\mathrm{ZnO} N W s$, fabricated by DEP over 3inch flexible PVC. (b) Image of the $4 \times 4$ UV PDs array under UV illumination. (c) Resulting PDs response as a function of the distance between the PD and the UV light source.

light power densities [53,54], hinder their prompt application in the field of UV PDs. In this regard, results obtained in this work confirm the validity of inorganic nanostructures such as $\mathrm{ZnO}$ NWs for near-future flexible UV PDs.

\section{Bendability of UV PDs Based on $\mathrm{ZnO} N W s$}

The performance of $\mathrm{ZnO}$ NWs based UV PDs consisting in the $2 \times 2$ array architecture (Fig. 2(d)) has been studied under dynamic bending conditions, with both compressive and tensile stress (Fig. 6). The dark current ( $\left.I_{\text {dark }}\right)$ of the four PDs integrated in the array, namely PD1-4, was measured under bending radius $(\theta)$ ranging between 5 and $27 \mathrm{~mm}$, i.e. extreme bending and almost flat conditions, respectively. For this study, the flexible UV PDs were mounted on a custom-made bending system, consisting of two-linear motors - controlled by Labview - and 3D printed platforms (see insets of Fig. 6)[56]. Fig. 6 shows the averaged $I_{\text {dark }}$ and standard deviation corresponding to PD1-4 (error bars). It may be noted from Fig. 6 that $I_{\text {dark }}$ is slightly affected by bending and exhibiting opposite behaviours under compressive and tensile bending. For example, the $I_{\text {dark }}$ of the device increases under compressive bending and a decrease with tensile bending. The slight change observed with respect to the flat conditions, could be due to two main factors: (i) a change in the inter-connections formed $\mathrm{Au}$ electrodes and output wires, or/and (ii) the change in the number of axial/longitudinal connection between NWs along the micro-fibre (see inset of Fig. 4(a)). The compressive stress (see top inset of Fig. 6) may increase the number of connections formed between NWs assembled with the shape of a micro-fibre (see inset of Fig. 4(a)) and thus result in a higher $I_{\text {dark }}$ with respect to the flat conditions. In contrast, the tensile stress can reduce the number of connections formed between NWs during DEP assembly, which can hinder the charge electric transport and eventually reduce the $I_{\text {dark, }}$, as observed in Fig. 6 . The selectivity to tensile and compressive bending conditions and sensitivity to different $\theta$, also make the fabricated device attractive for the development of mechanical sensors.

\section{E. Towards Large-area Fabrication of Flexible Dosimeters}

The scalability of our experimental procedure, in terms of fabrication of electrodes and DEP assembly of NWs over large areas (wafer scale), has been further demonstrated by expanding the size of the resulting flexible UV PDs array from $2 \times 2$ to $4 \times 4$, i.e. 16 PDs. Fig. 7 shows the image of $4 \times 4$ UV PDs based on $\mathrm{ZnO}$ NWs -fabricated by DEP- on top a 3-inch PVC flexible substrate. The $R_{\text {photo }}$ of each PD was acquired by a data acquisition (DAQ) board (from National Instruments) under different UV illuminations (Fig. 7(b)). The $4 \times 4$ UV PDs array exhibited high sensitivity to the intensity of received UV light. For example, the UV light source placed at different distances $(5$ and $20 \mathrm{~cm})$ resulted in the characteristic patterns shown in Fig. 7(c). The proximity of UV light source to PDs array was able to saturate some of the PDs, as can be noted from Fig. 7(c). The PDs at the centre of the substrate show 100\% response, whereas in far areas they exhibit less response.

\section{CONCLUSIONS}

In this work, we have grown $\mathrm{ZnO}$ NWs vertically aligned on c-plane sapphire by Au-assisted VLS mechanisms in CVT system. We thoroughly analysed the effect of parameters such as gas content, gas flow, and orientation of the substrate with respect the precursor gas direction, over resulting NWs aspect ratio and morphology. $\mathrm{ZnO} \mathrm{NWs}$ in solution were successfully assembled by DEP onto large-area flexible substrates. The scalability of above method has permitted the fabrication of $2 \times 2$ and $4 \times 4$ arrays of UV PDs over a 3 -inch flexible PVC substrate. The devices tested under different bending conditions, exhibited selectivity to compressive and tensile bending, as well as, sensitivity to bending radius. This also makes them suitable for mechanical/physical sensing. In addition, photoresponse of PDs array was characterized and modelled under different UV illuminations. The good agreement between the experimental results and the simulation/modelling would enable futuristic bendable integrated PDs along with readout circuits. All PDs showed a high sensitivity to UV light, with high $I_{\text {photo }} / I_{\text {dark }}$ ratios above $10^{3} \%$, fast response times $\left(\tau_{\mathrm{r}} \sim 12 \mathrm{~s}\right)$, high sensitivity to the intensity variations of the UV source. The high uniformity of the $R_{\text {photo }}$ measured from each PD, confirmed the reproducibility and reliability of our fabrication method to produce large-area UV sensitive wearable devices to monitor UV radiation e.g. in medical facilities, space expeditions, human activities, or health treatments. Future work will involve testing of presented devices in real conditions. To this end, we will also explore suitable packaging such as putting the PD arrays in a wristband, along with other components necessary for their operation.

\section{ACKNOWLEDGMENT}

This work was supported by Engineering and Physical Sciences Research Council (EPSRC) through Engineering Fellowship for Growth - PRINTSKIN (EP/M002527/1), and Centre for Doctoral Training in Intelligent Sensing and Measurement (EP/L016753/1). Authors are thankful to the support received for this work from James Watt Nanofabrication Centre (JWNC) and Electronic Systems Design Centre (ESDC). 


\section{REFERENCES}

[1] T. Sekitani, U. Zschieschang, H. Klauk, and T. Someya, "Flexible organic transistors and circuits with extreme bending stability," Nature materials, vol. 9, no. 12, p. 1015, 2010.

[2] M. Kaltenbrunner et al., "An ultra-lightweight design for imperceptible plastic electronics," Nature, vol. 499, no. 7459, p. 458, 2013.

[3] J. Viventi et al., "Flexible, foldable, actively multiplexed, high-density electrode array for mapping brain activity in vivo," Nature neuroscience, vol. 14, no. 12, p. 1599, 2011.

[4] W. Dang, L. Manjakkal, W. T. Navaraj, L. Lorenzelli, V. Vinciguerra, and R. Dahiya, "Stretchable wireless system for sweat $\mathrm{pH}$ monitoring," Biosens Bioelectron, vol. 107, pp. 192-202, Feb 102018.

[5] S. Xu et al., "Soft microfluidic assemblies of sensors, circuits, and radios for the skin," Science, vol. 344, no. 6179, pp. 70-74, 2014.

[6] S. Imani et al., "A wearable chemical-electrophysiological hybrid biosensing system for real-time health and fitness monitoring," Nature communications, vol. 7, p. 11650, 2016.

[7] K. Takei, W. Honda, S. Harada, T. Arie, and S. Akita, "Toward Flexible and Wearable Human - Interactive Health - Monitoring Devices," Advanced healthcare materials, vol. 4, no. 4, pp. 487-500, 2015.

[8] S. Patel, H. Park, P. Bonato, L. Chan, and M. Rodgers, "A review of wearable sensors and systems with application in rehabilitation," Journal of neuroengineering and rehabilitation, vol. 9, no. 1, p. 21, 2012.

[9] S. Gupta, W. T. Navaraj, L. Lorenzelli, and R. Dahiya, "Ultra-Thin Chips for High-Performance Flexible Electronics," NPJ Flexible Electronics, vol. DOI: 10.1038/s41528-018-0021-5, 2018.

[10] W. T. Navaraj, S. Gupta, L. Lorenzelli, and R. Dahiya, "Wafer Scale Transfer of Ultrathin Silicon Chips on Flexible Substrates for High Performance Bendable Systems," Advanced Electronic Materials, 4, 1700277, 2018

[11] K. Takei et al., "Nanowire active-matrix circuitry for low-voltage macroscale artificial skin," Nature Mater., vol. 9, no. 10, pp. 821-826, 2010.

[12] W. T. Navaraj et al., "Nanowire FET Based Neural Element for Robotic Tactile Sensing Skin," (in English), Frontiers in Neuroscience, vol. 11, no. 501, pp. 1-20, 2017.

[13] C. García Núñez, W. T. Navaraj, E. O. Polat, and R. Dahiya, "EnergyAutonomous, Flexible, and Transparent Tactile Skin," Advanced Functional Materials, vol. 27, p. 1606287, 2017, Art. no. 1606287.

[14] W. Dang, V. Vinciguerra, L. Lorenzelli, and R. Dahiya, "Printable stretchable interconnects," Flexible and Printed Electronics, vol. 2, no. 1, p. 013003, 2017.

[15] J. Kim et al., "Wearable smart sensor systems integrated on soft contact lenses for wireless ocular diagnostics," Nature Comm., vol. 8, p. 14997, 2017.

[16] R. S. Dahiya and M. Valle, Robotic tactile sensing: technologies and system. Springer Science \& Business Media, 2013.

[17] R. K. Sivamani, L. A. Crane, and R. P. Dellavalle, "The benefits and risks of ultraviolet tanning and its alternatives: the role of prudent sun exposure," Dermatologic clinics, vol. 27, no. 2, pp. 149-154, 2009.

[18] W. B. Grant, "An estimate of premature cancer mortality in the US due to inadequate doses of solar ultraviolet - B radiation," Cancer, vol. 94, no. 6, pp. 1867-1875, 2002.

[19] B. K. Armstrong and A. Kricker, "The epidemiology of UV induced skin cancer," Journal of photochemistry and photobiology B: Biology, vol. 63, no. 1-3, pp. 8-18, 2001.

[20] F. De Gruijl, "Skin cancer and solar UV radiation," European Journal of Cancer, vol. 35, no. 14, pp. 2003-2009, 1999.

[21] F. R. de Gruijl, H. J. van Kranen, and L. H. Mullenders, "UV-induced DNA damage, repair, mutations and oncogenic pathways in skin cancer," Journal of Photochemistry and Photobiology B: Biology, vol. 63, no. 13, pp. 19-27, 2001.

[22] J. H. Hoeijmakers, "DNA damage, aging, and cancer," New England Journal of Medicine, vol. 361, no. 15, pp. 1475-1485, 2009.

[23] Y. R. Helfrich, D. L. Sachs, and J. J. Voorhees, "Overview of skin aging and photoaging," Dermatology Nursing, vol. 20, no. 3, p. 177, 2008
[24] C. García Núñez, W. T. Navaraj, F. Liu, and R. Dahiya, "ZnO nanowires based flexible UV photodetectors for wearable dosimetry," in 2017 IEEE, SENSORS, 2017, vol. (DOI: 10.1109/ICSENS.2017.8234428), pp. 1-3.

[25] C. García Núñez, A. García Marín, P. Nanterne, J. Piqueras, P. Kung, and J. L. Pau, "Conducting Properties of Nearly Depleted ZnO Nanowire UV Sensors Fabricated by Dielectrophoresis.," Nanotechnology, vol. 24, p. 415702, 2013.

[26] C. Soci et al., "ZnO Nanowire UV Photodetectors with High Internal Gain," Nano Letters, vol. 7, no. 4, pp. 1003-1009, 2007.

[27] Ü. Özgür et al., "A comprehensive review of $\mathrm{ZnO}$ materials and devices," Journal of applied physics, vol. 98, no. 4, p. 11, 2005.

[28] C. García Núñez et al., "Enhanced fabrication process of zinc oxide nanowires for optoelectronics," Thin Solid Films, vol. 555, pp. 42-47, 2014.

[29] C. Chen and J. Zhu, "Bending strength and flexibility of $\mathrm{ZnO}$ nanowires," Applied physics letters, vol. 90, no. 4, p. 043105, 2007.

[30] J. Liu, N. Motta, and S. Lee, "Ultraviolet photodetection of flexible ZnO nanowire sheets in polydimethylsiloxane polymer," Beilstein journal of nanotechnology, vol. 3, p. 353, 2012.

[31] J. M. Wu, Y.-R. Chen, and Y.-H. Lin, "Rapidly synthesized ZnO nanowires by ultraviolet decomposition process in ambient air for flexible photodetector," Nanoscale, vol. 3, no. 3, pp. 1053-1058, 2011.

[32] C. García Núñez, A. F. Braña, N. López, and B. J. García, "GaAs Nanowires Grown by Ga-assisted Chemical Beam Epitaxy: Substrate Preparation and Growth Kinetics," Journal of Crystal Growth, vol. 430, pp. 108-115, 2015.

[33] C. García Núñez, W. T. Navaraj, F. Liu, D. Shakthivel, and R. Dahiya, "Large-Area Self-Assembly of Silica Microspheres/Nanospheres by Temperature-Assisted Dip-Coating," ACS Applied Materials \& Interfaces, vol. 10, no. 3, pp. 3058-3068, 2018.

[34] G. Shen, N. Dawahre, J. Waters, S. M. Kim, and P. Kung, "Growth, doping, and characterization of $\mathrm{ZnO}$ nanowire arrays," Journal of Vacuum Science \& Technology B, Nanotechnology and Microelectronics: Materials, Processing, Measurement, and Phenomena, vol. 31, no. 4, p. 041803, 2013.

[35] X. Liu, Y. Z. Long, L. Liao, X. Duan, and Z. Fan, "Large-scale integration of semiconductor nanowires for high-performance flexible electronics," ACS Nano, vol. 6, no. 3, pp. 1888-900, 2012.

[36] A. García Marín et al., "Continuous-flow system and monitoring tools for the dielectrophoretic integration of nanowires in light sensor arrays," Nanotechnology, vol. 26, no. 11, p. 115502, 2015.

[37] S. Raychaudhuri, S. A. Dayeh, D. Wang, and E. T. Yu, "Precise semiconductor nanowire placement through dielectrophoresis," Nano letters, vol. 9, no. 6, pp. 2260-2266, 2009.

[38] J. Suehiro, G. Zhou, and M. Hara, "Fabrication of a carbon nanotubebased gas sensor using dielectrophoresis and its application for ammonia detection by impedance spectroscopy," Journal of Physics D: Applied Physics, vol. 36, no. 21, p. L109, 2003.

[39] D. Wang, R. Zhu, Z. Zhou, and X. Ye, "Controlled assembly of zinc oxide nanowires using dielectrophoresis," Applied Physics Letters, vol. 90, no. 10, p. 103110, 2007.

[40] R. Dahiya, G. Gottardi, and N. Laidani, "PDMS residues-free micro/macrostructures on flexible substrates," Microelectronic Engineering, vol. 136, pp. 57-62, 2015.

[41] R. S. Dahiya, A. Adami, C. Collini, and L. Lorenzelli, "Fabrication of single crystal silicon micro-/nanostructures and transferring them to flexible substrates," Microelectronic Engineering, vol. 98, pp. 502-507, 2012.

[42] S. Khan, L. Lorenzelli, and R. Dahiya, "Flexible MISFET devices from transfer printed Si microwires and spray coating," IEEE Journal of the Electron Devices Society, vol. 4, no. 4, pp. 189-196, 2016.

[43] K. Fu, S. Chen, J. Zhao, and B. G. Willis, "Dielectrophoretic Assembly of Gold Nanoparticles in Nanoscale Junctions for Rapid, Miniature Chemiresistor Vapor Sensors," ACS Sensors, vol. 1, no. 4, pp. 444-450, 2016.

[44] F. Meng, H. Zheng, Y. Sun, M. Li, and J. Liu, "UV-activated room temperature single-sheet $\mathrm{ZnO}$ gas sensor," Micro \& Nano Letters, vol. 12, no. 10 , pp. 813-817, 2017. 
[45] A. Vilouras, H. Heidari, S. Gupta, and R. Dahiya, "Modeling of CMOS Devices and Circuits on Flexible Ultrathin Chips," IEEE Transactions on Electron Devices, vol. 64, no. 5, pp. 2038-2046, 2017.

[46] H. Heidari, N. Wacker, and R. Dahiya, "Bending induced electrical response variations in ultra-thin flexible chips and device modeling," Applied Physics Reviews, vol. 4, no. 3, p. 031101, 2017.

[47] J. Yao and G. Yang, "Flexible and High - Performance All - 2D Photodetector for Wearable Devices," Small, vol. 14, no. 21, p. 1704524, 2018.

[48] M. Qiu et al., "Visualized UV Photodetectors Based on Prussian Blue/TiO2 for Smart Irradiation Monitoring Application," Advanced Materials Technologies, vol. 3, no. 2, p. 1700288, 2018.

[49] P. Hu et al., "Highly responsive ultrathin GaS nanosheet photodetectors on rigid and flexible substrates," Nano letters, vol. 13, no. 4, pp. 16491654,2013

[50] Q. Zheng, J. Huang, S. Cao, and H. Gao, "A flexible ultraviolet photodetector based on single crystalline $\mathrm{MoO} 3$ nanosheets," Journal of Materials Chemistry C, vol. 3, no. 28, pp. 7469-7475, 2015.

[51] P. Sahatiya, S. S. Jones, and S. Badhulika, "2D MoS 2-carbon quantum dot hybrid based large area, flexible UV-vis-NIR photodetector on paper substrate," Applied Materials Today, vol. 10, pp. 106-114, 2018.

[52] Z. Lou, L. Li, and G. Shen, "High-performance rigid and flexible ultraviolet photodetectors with single-crystalline $\mathrm{ZnGa} 2 \mathrm{O} 4$ nanowires," Nano Research, vol. 8, no. 7, pp. 2162-2169, 2015.

[53] S. Chen, C. Teng, M. Zhang, Y. Li, D. Xie, and G. Shi, "A Flexible UVVis - NIR Photodetector based on a Perovskite/Conjugated - Polymer Composite," Advanced Materials, vol. 28, no. 28, pp. 5969-5974, 2016.

[54] X. Hu et al., "High - Performance Flexible Broadband Photodetector Based on Organolead Halide Perovskite," Advanced Functional Materials, vol. 24, no. 46, pp. 7373-7380, 2014.

[55] S. Bai, W. Wu, Y. Qin, N. Cui, D. J. Bayerl, and X. Wang, "High performance integrated $\mathrm{ZnO}$ nanowire UV sensors on rigid and flexible substrates," Advanced Func. Mater., vol. 21, no. 23, pp. 4464-4469, 2011.

[56] W. Dang, L. Manjakkal, W. T. Navaraj, L. Lorenzelli, V. Vinciguerra, and R. Dahiya, "Stretchable wireless system for sweat pH monitoring," Biosensors and Bioelectronics, vol. 107, pp. 192-202, 2018.

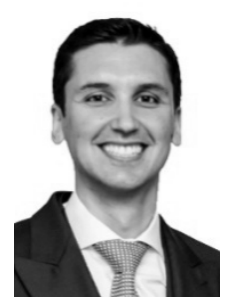

Dr. Carlos García Núñez received his B.S. degree in Physics in 2009, his M.S. degree in Advanced Materials and Nanotechnology in 2010, and his $\mathrm{PhD}$ degree in Physics in 2015, in the Department of Applied Physics from Universidad Autónoma de Madrid, Spain. Since 2015, he has been Postdoctoral Researcher with the School of Engineering, University of Glasgow (UK). He has authored over 30 research articles, 1 book chapter, and 1 patent. His research interest includes synthesis and characterization of semiconductor thin films and nanomaterials such as nanowires and 2D structures, for the development of optoelectronics, photovoltaics, electronics, sensors, and wearable systems. His research also comprises the integration of above nanomaterials by contactprinting, dielectrophoresis and optoelectronic tweezing.

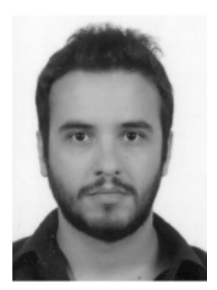

Anastasios Vilouras (S'16) received his B.Sc. degree in Physics from Aristotle University of Thessaloniki, Greece in 2014, and his M.Sc. degree on Bioelectronics and Biosensors from University of Edinburgh, UK in 2015. He joined the Centre for Doctoral Training in Intelligent Sensing and Measurement (CDT-
ISM) of University of Glasgow, UK in 2016, where he is currently working towards his Ph.D. degree in Bendable Electronics and Sensing Technologies (BEST) group focusing mainly on CMOS-based bendable sensors for chemical and biochemical measurements.

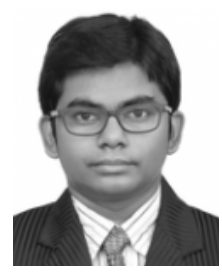

William Taube Navaraj received his B.E. degree in Electronics and Communication Engineering from Anna University in 2009 and M. Tech. in Advanced Semiconductor Electronics from Academy of Scientific and Innovative Research in 2011. Before joining University of Glasgow for his $\mathrm{PhD}$, he was a Scientist in Sensors and Nanotechnology Group at CSIRCentral Electronics Engineering Research Institute, Pilani.

His current research interests include flexible micro/nano/optoelectronics, assistive robotics and flexible biomedical electronic systems. He has won CSIR-QHS Fellowship, best paper awards in international conferences, Department Topper during Masters' Degree, Child scientist award from NCSTCDST, District Topper in Higher Secondary Exams, Anna Award from Govt. of Tamil Nadu and several prizes in various engineering and robotics projects. He is a member of IEEE-UK, IET-UK, associate member of IE (India), and life fellow of OSI.

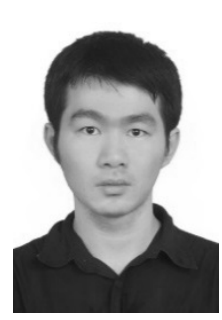

Fengyuan Liu received the B.S. degree in Physics in 2012 and M.S. degree in Electronic Engineering in 2015, from Nanjing University (China). He is currently pursuing his Ph.D. degree in the School of Engineering from University of Glasgow (UK). His research interests include flexible electronic devices and circuits based on nano-materials (especially quasi-1D nanowires and quasi-2D graphene), printing and $3 \mathrm{D}$ electronics.

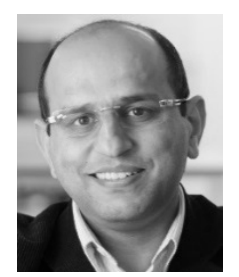

Ravinder Dahiya ( $\left.\mathrm{S}^{\prime} 05, \mathrm{M}^{\prime} 09, \mathrm{SM}^{\prime} 12\right)$ is Professor of Electronics and Nanoengineering in the University of Glasgow, U.K. He is the leader of Bendable Electronics and Sensing Technologies (BEST) research group. His group conducts fundamental and applied research in the multidisciplinary fields of flexible and printable electronics, tactile sensing, electronic skin, robotics and wearable systems. He has authored over 200 research articles, 4 books, and 11 patents. He has led several international projects. He is a Distinguished Lecturer of the IEEE Sensors Council and is serving on the editorial boards of the Scientific Report and IEEE SENSORS JOURNAL. He is the Technical Program cochair of IEEE Sensors 2017 and IEEE Sensors 2018. He holds the prestigious EPSRC Fellowship and received in past the Marie Curie Fellowship and Japanese Monbusho Fellowship. In 2016, he got the Microelectronic Engineering Young Investigator Award and the 2016 Technical Achievement Award from the IEEE Sensors Council. 